Reduced order models describing

hydraulic and mechanical apertures of cement-fracture as a function of fracture roughness, confining pressure and offset.

S. D. C. Walsh

October 4, 2012 
This document was prepared as an account of work sponsored by an agency of the United States government. Neither the United States government nor Lawrence Livermore National Security, LLC, nor any of their employees makes any warranty, expressed or implied, or assumes any legal liability or responsibility for the accuracy, completeness, or usefulness of any information, apparatus, product, or process disclosed, or represents that its use would not infringe privately owned rights. Reference herein to any specific commercial product, process, or service by trade name, trademark, manufacturer, or otherwise does not necessarily constitute or imply its endorsement, recommendation, or favoring by the United States government or Lawrence Livermore National Security, LLC. The views and opinions of authors expressed herein do not necessarily state or reflect those of the United States government or Lawrence Livermore National Security, LLC, and shall not be used for advertising or product endorsement purposes.

This work performed under the auspices of the U.S. Department of Energy by Lawrence Livermore National Laboratory under Contract DE-AC52-07NA27344. 


\title{
Reduced order models describing hydraulic and mechanical apertures of cement-fracture as a function of fracture roughness, confining pressure and offset.
}

\author{
Stuart D.C. Walsh
}

Long-term sequestration of carbon-dioxide is intimately linked to wellbore integrity, as wellbores present an obvious vertical conduit for subsurface transmission of $\mathrm{CO} 2$ and $\mathrm{CO} 2$-rich brines. Understanding the movement of these fluids along cement fractures and interfaces is of particular importance, as these pathways offer potential escape routes around seals and out of the wellbore environment.

The high degree of heterogeneity in subsurface and well conditions, combined with an absence of knowledge about environmental factors, implies that these systems are often best investigated using uncertainty quantification and risk analysis techniques. However, to be effective such methods require lightweight models for rapid quantification of multiple realizations - full-scale high fidelity simulations are simply too computationally expensive.

Hence, there is a need for reduced order models, derived from more detailed simulations, that accurately reproduce the relevant system response over a range of appropriate input parameters. The following report details the development of such a reduced order model to describe the behavior of fractures in wellbore cement.

The model has been developed to provide input for a larger-scale model representing the large-scale geomechanical behavior of the wellbore-system [9]. The larger-scale model has been developed in a modular fashion with several avenues for user modification - including the ability to specify the effect of stress/deformation on changes to the permeability tensor. It is to this permeability module that the reduced order model described in this paper will be coupled.

Specifically, the reduced order model describes the response of hydraulic and mechanical aperture in cement fractures as a function of fracture roughness, confining pressure, offset and fluid volume fraction. In Section 1, we outline the underlying numerical model from which the reduced order model is derived. The underlying high-fidelity model is used to conduct a series of simulations, the results of which are summarized in Section 2. The reduced order model is then fitted to these results, as presented in Section 3. Conclusions and future 


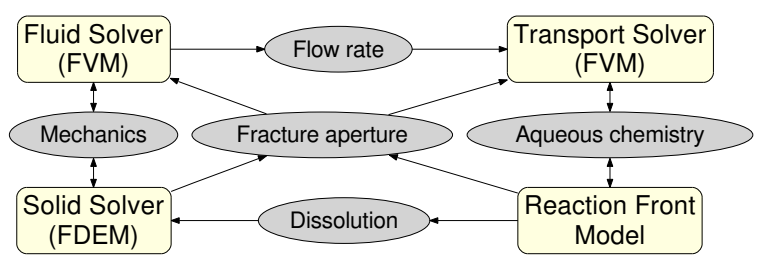

Figure 1: Flow-chart of the solvers employed to simulate fracture growth and healing and the processes by which they are coupled.

work are discussed in Section 4.

\section{Model Description}

Portland cement exposed to carbon-dioxide-rich brine undergoes a series of diffusion limited reactions that result in the formation of distinctive reaction fronts adjacent to the cement-brine interface. The development of these reaction fronts may in turn trigger fracture growth or sealing, as a result of linked geochemical, hydrodynamic and geomechanical processes acting on the interface. Simulation of these varied processes requires integration of several distinct physical models in a common numerical framework.

- A model to capture the development of distinctive reaction zones within the cement and host-rock.

- A fluid-mechanics solver to capture flow along the fracture.

- A transport solver to model advection, diffusion and reaction of aqueous chemical species.

- A solid-mechanics solver to reproduce the host rock response to changes in pore and confining pressure.

We have developed independent numerical models for each of the processes listed above, and combined them in GEOS (formerly GPAC) - an open-source computational framework for multi-scale multi-physics simulations with a focus on the geosciences, which is under development at Lawrence Livermore National Laboratory $[17,22]$. The separate solvers, and the processes by which they are coupled is illustrated in Figure 1.

Ultimately, we plan the development of reduced order models for fracture flow involving all of these process. However, the preliminary model described in this report is concerned primarily with the description of the effect of the coupling between solid-deformation and flow along the fracture. Deformation of the cement and host rock is modeled using an implicit Lagrange discrete finite element solver. The deformation solver is coupled to the flow solver via the fluid pressure applied to the fracture surface. In addition, the fracture aperture is 
controlled by the relative positions of the deformed surfaces of the solid body. This provides the ability to simulate the effects of confining pressures on the flow characteristics of a sample, as well as simulating the effect of the fluid pressure on the aperture. Flow within the fractured regions are modeled with a cell-centered finite difference approach, in which the fracture is represented as a set of two-dimensional finite volume elements embedded in a three-dimensional volume. A similar approach, used to simulate fluid flow for hydraulic fracturing simulations, is described in [6]. The fracture width is determined by an aperture thickness ascribed to each element in the two dimensional surface.

The fracture surface is created using a routine designed to produce a prescribed surface roughness given by the JRC scale $[1,7]$. A comparison between the original JRC surfaces proposed by Barton and the surfaces created by the fracture generation routine is given in Figure 2. Although the fractures shown in Figure 2 are one-dimensional, the simulations used to create the reduced order model employ two-dimensional fractures in three dimensional solid bodies with periodic boundary conditions are enforced along the fracture boundaries. As JRC values are somewhat qualitative and several techniques have been proposed for their estimation, the surface roughness is also tracked using the more quantitative $Z 2$ measure [20,23]. The dimensionless Z2 roughness measure originally proposed by Myers [10] is defined as:

$$
Z 2=\left[\frac{1}{L} \int_{0}^{L}\left(\frac{d y}{d x}\right)^{2} d x\right]^{1 / 2}
$$

where $y(x)$ describes the profile of the fracture surface. In practice, the $Z 2$ value is determined from a discretization of the surface profile

$$
Z 2=\left[\frac{1}{L} \sum \frac{\left(y_{i+1}-y_{i}\right)^{2}}{x_{i+1}-x_{i}}\right]^{1 / 2},
$$

where $x_{i}$ and $y_{i}$ are the coordinates of the fracture surface, typically taken at regular sampling intervals, $\Delta x_{i}=x_{i+1}-x_{i}$. Real fractures are multi-fractal in nature, implying that roughness parameters like the $Z 2$ measure are contingent upon the scale at which they are measured. One method of overcoming this sensitivity is to fix the sampling interval - however, the choice of interval is somewhat arbitrary. Yu and Vayssade [23] derived empirical equations relating $Z 2$ and JRC values using sampling intervals of $1.0 \mathrm{~mm}, 0.5 \mathrm{~mm}$ and $0.25 \mathrm{~mm}$. For consistency with their results and to fix a measurement scale for comparison with natural materials, the sampling frequency used to determine the $\mathrm{Z} 2$ value in our simulations is set to $0.25 \mathrm{~mm}$.

The fracture aperture is generated by separating the two mated fracture surfaces that split the block, imposing a shear displacement, and then applying the confining pressure to the block exterior. Due to the enforced periodicity of the fracture surface, the aperture distribution is also periodic. For simplicity, the two fracture surfaces are treated as elastic bodies, hence the effects of asperity damage are ignored in this calculation. 
a)

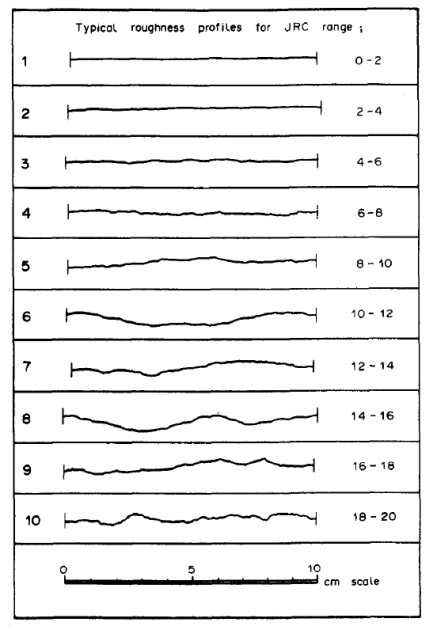

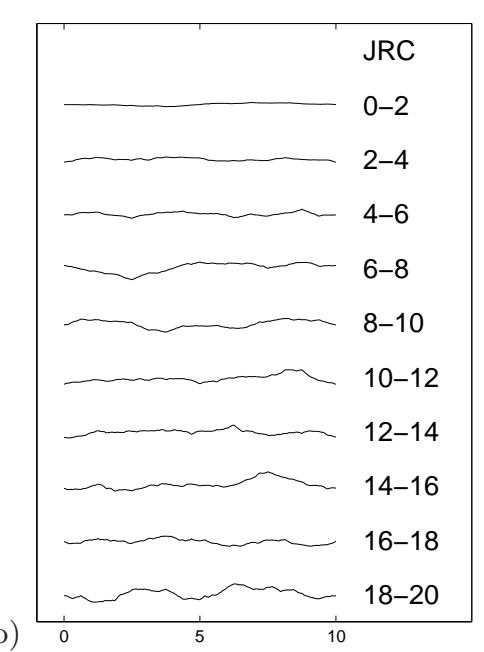

)

Figure 2: Comparison between a) surface roughness profiles used in the JRC roughness scale and b) corresponding roughness profiles produced with the surface generation routine.

After the fractures have been generated, both their mechanical and hydraulic apertures are recorded for the reduced order model. The mechanical aperture $h_{m}$ is given by the mean aperture for the fracture

$$
h_{m}=\frac{1}{l_{x} l_{y}} \int h(x, y) d x d y,
$$

where $l_{x}$ and $l_{y}$ are the length and width of the fracture and $h(x, y)$ is the fracture aperture at a given location.

The hydraulic aperture of the fracture is determined by applying a pressure gradient along the fracture and measuring the resulting flux. Periodic boundary conditions are applied in the transverse direction to the flow. For a fluid flowing between two parallel plates, the net volume flux is related to the aperture by the so-called "cubic law"

$$
Q=\frac{|\nabla P| h^{3} w}{12 \mu},
$$

where $\mu$ is the viscosity, $h$ is the aperture between the plates, $w$ is the width of the plates, and $\nabla P$ is the pressure gradient. Comparing this equation with Darcy's law,

$$
Q=\kappa A \frac{|\nabla P|}{\mu},
$$

where the area $A=h w$ in the parallel plate system, yields the following expression for the permeability, $\kappa$ :

$$
\kappa=\frac{h^{2}}{12},
$$




$\begin{array}{ll}\text { Parameter } & \text { Range } \\ \text { JRC } & 1-16 \\ \text { Confining Pressure } & 1-27 \mathrm{MPa} \\ \text { Pore Pressure/Confining Pressure Ratio } & 0-1 \\ \text { Shear displacement in direction of flow } & 0-10 \mathrm{~mm} \\ \text { Shear displacement perpendicular to flow } & 0-10 \mathrm{~mm} \\ \text { Bulk Modulus } & 7.5-15 \mathrm{GPa}\end{array}$

Table 1: Simulation parameter ranges.

while the transmissivity, $T$, is related to the parallel plate aperture by

$$
T=\frac{w h^{3}}{12} .
$$

In natural systems, the aperture is not constant but varies over the fracture, and due to the geometric changes in the fracture surface, the mechanical aperture is not appropriate for describing fracture transmissivity. Instead, a hydraulic aperture is introduced, which (to maintain continuity with the parallel plate system) is defined as:

$$
h_{h}=\sqrt[3]{\frac{12 T}{w}},
$$

where transmissivity in equation 8 is related to the flux $Q$ by

$$
T=\frac{Q \mu}{|\nabla P|} .
$$

\section{Simulation results}

To derive the reduced order model, simulations were conducted of fractured blocks of dimensions $10 \mathrm{~cm} \times 10 \mathrm{~cm} \times 2 \mathrm{~cm}$. Each block was divided into 200,000 cells with $\sim 1 \mathrm{~mm}$ dimension on each side. A typical mesh with the fracture surface highlighted (apertures shown in color) is given in Figure 3.

In total 13,500 simulations were conducted for 1350 distinct sets of input sample parameters (i.e. 10 simulations per sample point). Sample selection was based on a quasi-Monte Carlos sampling method (LPTAU) using PSUADE - a software toolkit to facilitate uncertainty quantification [19]. The ranges for each of the simulation parameters are summarized in Table 1, and plots of the input sample distributions are shown in Figure 4.

Plots comparing output parameters - hydraulic aperture and mechanical aperture - are shown in Figure 5. Two things are immediately obvious - 1) consistent with experimental observation, the hydraulic aperture is generally less than or equal to the mechanical aperture, and 2) perhaps contrary to expectation, in a few cases the hydraulic aperture is greater than the mechanical aperture. Both observations may be explained as follows. 


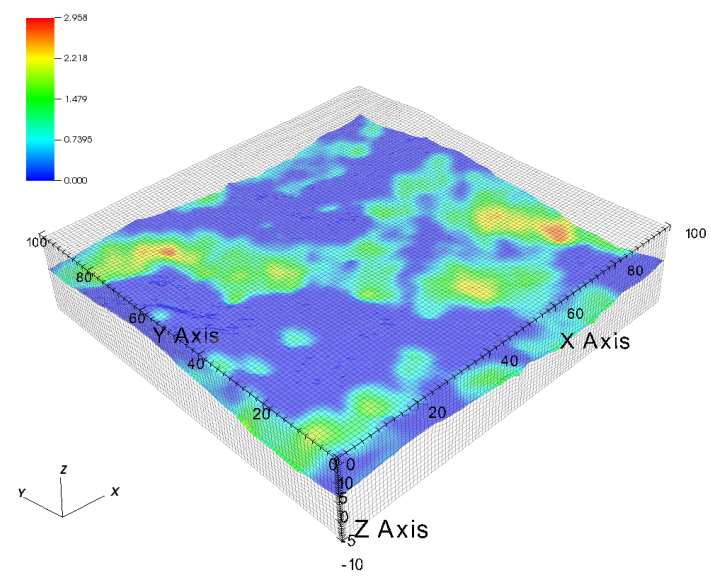

Figure 3: Typical mesh used in a single simulation. The fracture surface is highlighted showing the aperture distribution.

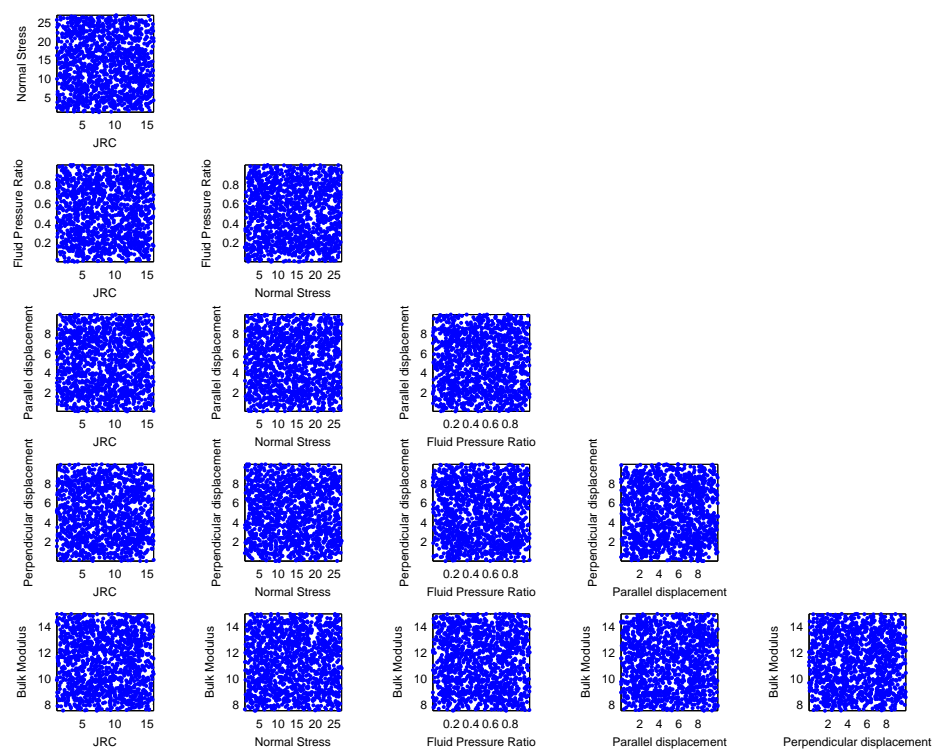

Figure 4: Plots of simulation input parameter combinations (each point represents 10 simulations). 


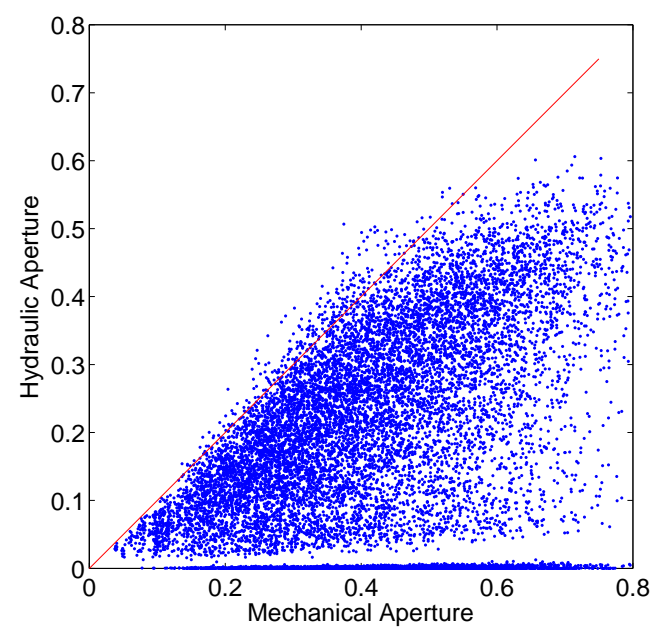

Figure 5: Plots of simulation output comparing mechanical and hydraulic aperture (each point represents 1 simulation). Solid line indicates equality between the two aperture measures.

Hydraulic aperture is defined as the aperture between two parallel plates with a hydraulic transmissivity equivalent to that of the fracture, whereas the mechanical aperture represents the volume of fluid it contains. In general, the tortuosity of the connected flow-pathways within a fracture and the constrictions along those pathways both act to reduce the transmissivity of the fracture, therefore in most cases the mechanical aperture is greater than the hydraulic aperture. However, flow between two parallel plates is not the most efficient means of transmitting fluid for a given average aperture. It is more effective, for example, to concentrate the fluid along a narrow pipe than between two parallel plates. Thus in a few cases where the fracture consists of a relatively straight and even flow-path aligned in the direction of the applied pressure gradient the hydraulic aperture is greater than the mechanical aperture.

Interestingly, the few cases where the hydraulic aperture is greater than the mechanical aperture occur when the fracture aperture is translated perpendicular to the direction of the applied pressure gradient. This is due to the fact that the apertures created between the two correlated fracture surfaces are generally aligned perpendicular to the relative displacements of the two surfaces. Roughly speaking, shifting the surfaces generates fractures apertures that are correlated with the gradient of the fracture in the direction of the shift. Thus larger apertures are generated along contours aligned perpendicular to the relative motion of the two fracture surfaces, resulting in preferential flow pathways aligned along those directions. For the same reason, flow in the transverse direction is hindered. This result stresses the need to consider anisotropic permeability models when simulating the fracture flow. 

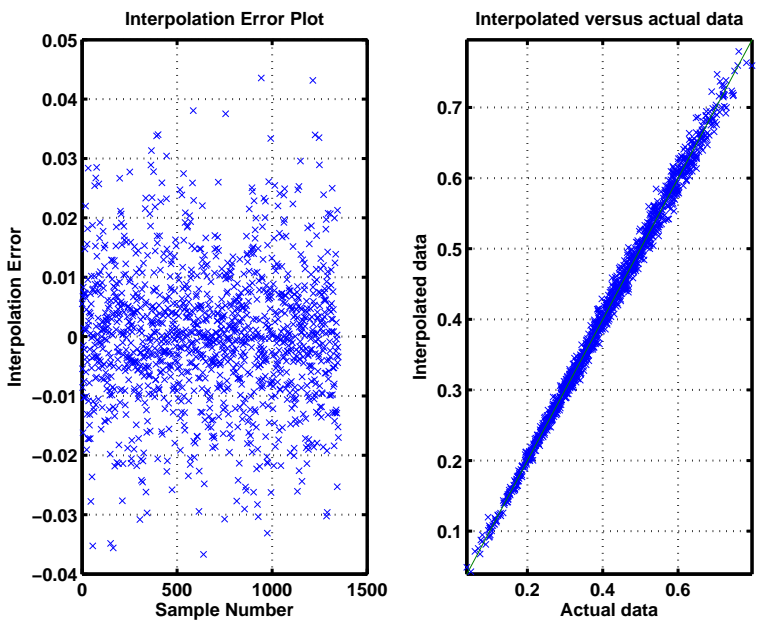

Figure 6: Mechanical aperture interpolation error and model/data comparison for Run Three: $R^{2}=0.99$. Each point represents ten simulations.

\section{Reduced order models}

Reduced order models were fitted to the results of the simulations described in Section 2 using the PSUADE software toolkit. Comparisons between the results of the simulation runs and the fitted results from the reduced order model are given in Figure 6 and Figure 7. An extremely good fit was obtained for the reduced order model for the mechanical aperture $\left(R^{2}=0.99\right)$, reflecting the fact that the mechanical aperture is relatively insensitive to variations in the realizations of the fracture surface. The hydraulic aperture, which is influenced by both bottle-necks in the fracture flow pathways and long range correlations in the fracture aperture distribution, showed greater variation $\left(R^{2}=0.87\right)$.

As many models relate both hydraulic and mechanical apertures, and the mechanical aperture serves as a rough bound on the hydraulic aperture, a second reduced order model was also developed that incorporated the mechanical aperture in the fitting parameters (Figure 8). This resulted in a slightly higher $R^{2}$ value $\left(R^{2}=0.89\right)$ though this is most likely due to the expansion of the number of fitting parameters rather than an improvement in the overall "goodness" of the fit.

The Lewis et al. [9] wellbore-scale model employs the concept of relative permeability to describe the relative motion of the separate components in fluid mixtures (as would be encountered for example in a supercritical $\mathrm{CO}_{2} /$ brine mixture). To capture this behavior, a relative permeability module has been included with the reduced order model for the hydraulic conductivity.

Relative permeability measures the ratio of the effective permeability of a single phase in a binary fluid, $\kappa_{e f f}$, relative to the permeability of the fully 

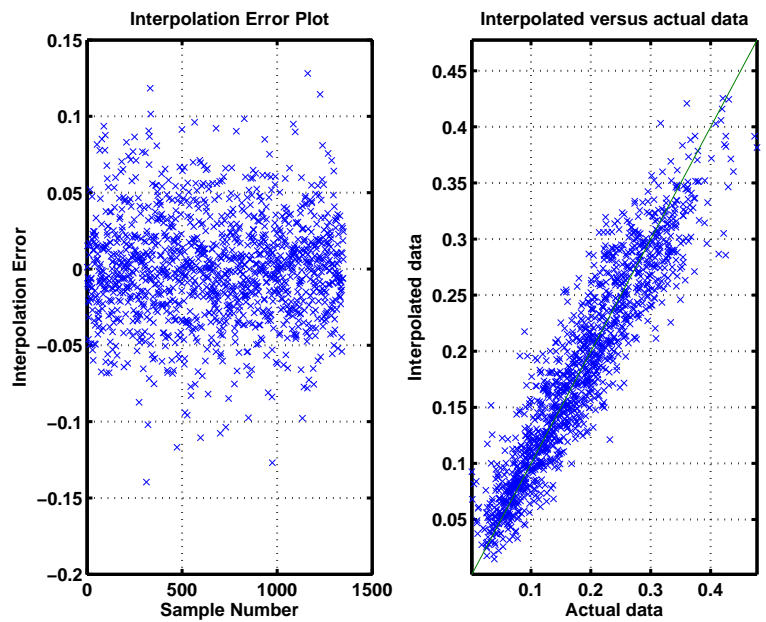

Figure 7: Hydraulic aperture interpolation error and model/data comparison for Run Three: $R^{2}=0.87$. Each point represents ten simulations.
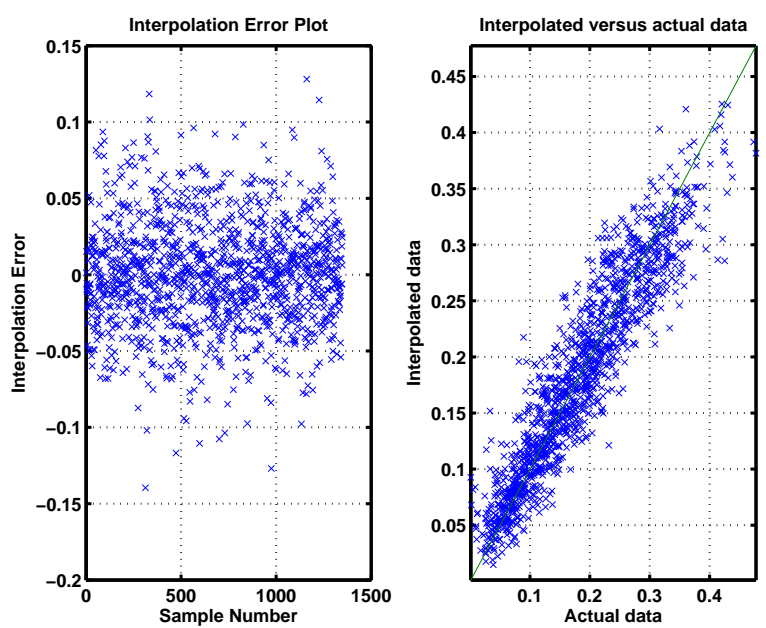

Figure 8: Hydraulic aperture interpolation error and model/data comparison using mechanical aperture and removing the outlier: $R^{2}=0.89$. Each point represents ten simulations. 
saturated phase, $\kappa$.

$$
\kappa_{e f f}=k_{r} \kappa .
$$

There is currently little consensus on the correct functional form for relative permeability in fractures. What is clear is that relative permeability is dependent on a range of factors including: the rate of flow, aperture anisotropy, flow history (i.e. imbibition vs drainage), and capillary forces. The latter is particularly problematic for CO2/brine binary-fluid mixtures - as brine is generally the wetting fluid for most rock-types, but the mixed fluid may become neutrally wetting or CO2-wetting when in contact with oil-wet rocks [4]. Finally, the wetting angle is further affected by small-scale heterogeneity, such as local minerology and micro-roughness.

Nevertheless, the relative permeability can be bounded, albeit fairly conservatively, by the linear or ' $\mathrm{X}$ ' model:

$$
\begin{aligned}
k_{r_{w}} & =S_{w} \\
k_{r_{n} w} & =S_{n w}=1-S_{w} .
\end{aligned}
$$

This model assumes that there is no phase interference in the flow - essentially treating the two fluids as flowing through adjacent, non-blocking pathways. While early measurements of relative permeability in parallel plate tests reported agreement with the linear model [16], and some evidence was found supporting this model in steam-water flows from geothermal fields [15], since then several experiments conducted on parallel and roughened plates have reported contradictory results $[3,12,13]$. Nevertheless, such a model may be more appropriate for neutrally wetting surfaces or fractures with large apertures, where capillary forces are less dominant.

There is some evidence to suggest that relative permeability in fractures is somewhat better approximated by the Corey model $[2,3]$.

$$
\begin{aligned}
k_{w} & =S^{* 4} \\
k_{n w} & =\left(1-S^{*}\right)^{2}\left(1-S_{w}^{* 2}\right),
\end{aligned}
$$

where $S^{*}$ is the normalized wetting saturation, defined as

$$
S^{*}=\left(S_{w}-S_{r w}\right) /\left(1-S_{r w}-S_{r n w}\right),
$$

in which $S_{r w}$ is the residual saturation of the wetting phase, while $S_{r n w}$ is the residual saturation of the non-wetting phase. Diomampo [3] fitted values of the residual saturations in for water-gas mixtures in fractures to the related Honarpour residual permeability equation [5]:

$$
\begin{aligned}
k_{w} & =\left(S^{*}\right)^{n_{w}} \\
k_{n w} & =\left(S^{\dagger}\right)^{n_{n w}},
\end{aligned}
$$

where $S^{\dagger}$ is the normalized non-wetting saturation

$$
S^{\dagger}=\left(S_{n w}-S_{r n w}\right) /\left(1-S_{r w}-S_{r n w}\right),
$$


and the exponents $n_{w}$ and $n_{n w}$ are empirical constants. For drainage experiments between parallel plates, Diomampo's fitted exponents were close to the exponents in the Corey model, and while the same was not true of the imbibition experiments, measured relative permeabilities for both the rough and parallel plate test followed the trend predicted by the Corey model. The fitted values of $S_{r w}$ were around $0.08-0.14$, while $S_{r n w}$ was 0 for drainage and $0.32-0.5$ for imbibition.

Elsewhere, flow along fractures has been considered analogous to highly permeable media with intergranular porosity [14], leading to the adoption of van Genuchten functions [21] to describe relative permeability:

$$
k_{w}=\sqrt{S^{*}}\left(1-\left(1-\left[S^{*}\right]^{1 / \lambda}\right)^{\lambda}\right)^{2},
$$

where $\lambda$ is related to the pore size distribution. Using a similar approach to van Genuchten, Parker and co-workers $[8,11]$ derived an expression for the relative permeability of the non-wetting phase:

$$
k_{n w}=\sqrt{1-S^{*}}\left(1-\left[S^{*}\right]^{1 / \lambda}\right)^{2 \lambda} .
$$

Despite the differences between the various models and potential for variation due to changes in rock/fluid interactions, the relative permeability is not necessarily a driving factor in binary-fluid behavior over system scales. For example, studies of geothermal fields showed little variation in the system response as a function of the relative permeability model employed [18]. In addition the linear model, while conservative, provides a strict upper bound on the relative permeability of both phases. Thus it is recommended that the linear and Corey models be used to gauge the system sensitivity to the relative permeability, using the linear model to bracket the maximum flux along the fracture.

\section{Conclusions and Future Work}

This report details the derivation of a reduced order model describing hydraulic and mechanical aperture in cement fractures, as a function of function of fracture roughness, confining pressure and offset. The reduced order model was fitted to results from over thirteen thousand high fidelity simulations. A very good fit was obtained for the reduced order model for the mechanical aperture $\left(R^{2}=0.99\right)$. The reduced order model for the hydraulic aperture is slightly less good $\left(R^{2}=\right.$ 0.87 ), due to the sensitivity of this parameter to preferential flow pathways and local bottlenecks. Nevertheless, the fit could be improved by including the mechanical aperture in the reduced order model $\left(R^{2}=0.89\right)$.

Additional subroutines describing the effect of the relative permeability on the model have been included in the reduced order model. Unfortunately, there is little consensus on the correct relative permeability model for fractures, and all appear to be susceptible to local variations in surface properties. In place of a definitive model, it is recommended that the linear and Corey models be used to gauge system sensitivity to the form of the relative permeability function. 


\section{References}

[1] N. Barton and V. Choubey. The shear strength of rock joints in theory and practice. Rock Mechanics and Rock Engineering, 10:1-54, 1977. 10.1007/BF01261801.

[2] A.T. Corey. The interrelation between gas and oil relative permeabilities. Producers monthly, 19(1):38-41, 1954.

[3] G. Diomampo, C.Y. Chen, K. Li, and R.N. Horne. Relative permeability through fractures. In Proc. $2^{\text {ry }}$ th Workshop on Geothermal Reservoir Engineering, pages 28-30, 2001.

[4] D.N. Espinoza and J.C. Santamarina. Water-CO2-mineral systems: Interfacial tension, contact angle, and diffusionimplications to $\mathrm{CO} 2$ geological storage. Water resources research, 46(7):W07537, 2010.

[5] M. Honarpour, LF Koederitz, and A. Harvey. Empirical equations for estimating two-phase relative permeability in consolidated rock. Journal of Petroleum Technology, 34(12):2905-2908, 1982.

[6] S.M. Johnson and J.P. Morris. Modeling hydraulic fracturing for carbon sequestration applications. In The 43rd US Rock Mechanics Symposium and the 4th US-Canada Rock Mechanics Symposium, pages ARMA 09-30, Asheville, NC, USA, 2009. American Rock Mechanics Association.

[7] Y.-H. Lee, J.R. Carr, D.J. Barr, and C.J. Haas. The fractal dimension as a measure of the roughness of rock discontinuity profiles. International Journal of Rock Mechanics and Mining Sciences 8 Geomechanics Abstracts, 27(6):453-464, 1990 .

[8] RJ Lenhard and JC Parker. A model for hysteretic constitutive relations governing multiphase flow, 2. permeability-saturation relations. Water Resour. Res, 23(12):2197-2206, 1987.

[9] Kayla Lewis, George Zyvoloski, Sharad Kelkar, and J. William Carey. Coupled stress and flow along interfaces in the wellbore environment in relation to co2 sequestration. In The 46th US Rock Mechanics Symposium, Chicago, IL, ARMA 12, page in press, 2012.

[10] N.O. Myers. Characterization of surface roughness. Wear, 5(3):182-189, 1962.

[11] JC Parker, RJ Lenhard, and T. Kuppusamy. A parametric model for constitutive properties governing multiphase flow in porous media. Water Resour. Res, 23(4):618-624, 1987.

[12] P. Persoff and K. Pruess. Two-phase flow visualization and relative permeability measurement in natural rough-walled rock fractures. Water Resour. Res, 31(5):1175, 1995. 
[13] P. Persoff, K. Pruess, and L. Myer. Two-phase flow visualization and relative permeability measurement in transparent replicas of rough-walled rock fractures. Lawrence Berkeley Lab, 1991.

[14] K. Pruess. On water seepage and fast preferential flow in heterogeneous, unsaturated rock fractures. Journal of contaminant hydrology, 30(3-4):333$362,1998$.

[15] K. Pruess and YW Tsang. On two-phase relative permeability and capillary pressure of rough-walled rock fractures. Water Resources Research, 26(9):1915-1926, 1990.

[16] ES Romm. Flow characteristics of fractured rocks. Nedra, Moscow, 283, 1966.

[17] Randolph Settgast, Scott Johnson, Pengcheng Fu, Stuart D.C. Walsh, and Frederick Ryerson. Simulation of hydraulic fracture networks in three dimensions. In PROCEEDINGS, Thirty-Seventh Workshop on Geothermal Reservoir Engineering, pages SGP-TR-194, Stanford University, Stanford, California, January 30 - February 12012.

[18] M.L. Sorey, M.A. Grant, and E. Bradford. Nonlinear effects in twophase flow to wells in geothermal reservoirs. Water Resources Research, 16(4):767-777, 1980.

[19] CH Tong. The psuade software library. Lawrence Livermore National Laboratories UCRL UCRL-WEB-211742. Web site: http://www. llnl. gov/CASC/uncertainty_-quantification/\# psuade, 253, 2005.

[20] R. Tse and D.M. Cruden. Estimating joint roughness coefficients. In International Journal of Rock Mechanics and Mining Sciences $\&$ Geomechanics Abstracts, volume 16, pages 303-307. Elsevier, 1979.

[21] M.T. Van Genuchten. A closed-form equation for predicting the hydraulic conductivity of unsaturated soils. Soil Science Society of America Journal, 44(5):892-898, 1980.

[22] S. D. C. Walsh, W. L. Du Frane, Y. Sholokhova, R. Settgast, S. M. Johnson, and S. Carroll. Chemo-mechanical permeability evolution in wellborecement fractures exposed to carbon-dioxide-rich brines. In The 46th US Rock Mechanics Symposium, Chicago, IL, ARMA, pages 12-426, 2012.

[23] X. Yu and B. Vayssade. Joint profiles and their roughness parameters. In International journal of rock mechanics and mining sciences 85 geomechanics abstracts, volume 28, pages 333-336. Pergamon, 1991. 